\title{
Nuclear coalescence from correlation functions
}

\author{
Kfir Blum* \\ Theory Department, CERN, CH-1211 Geneve 23, Switzerland \\ and Weizmann Institute, Department of Particle Physics and Astrophysics, Rehovot 7610001, Israel \\ Masahiro Takimoto \\ Weizmann Institute, Department of Particle Physics and Astrophysics, Rehovot 7610001, Israel
}

(Received 26 January 2019; published 18 April 2019)

\begin{abstract}
We derive a simple formula relating the cross section for light cluster production (defined via a coalescence factor) to the two-proton correlation function measured in heavy-ion collisions. The formula generalizes earlier coalescence-correlation relations found by Scheibl \& Heinz and by Mrowczynski for Gaussian source models. It motivates joint experimental analyses of Hanbury-Brown-Twiss (HBT) and cluster yield measurements in existing and future data sets.
\end{abstract}

DOI: 10.1103/PhysRevC.99.044913

\section{INTRODUCTION}

The Large Hadron Collider (LHC) made available a diverse data set of production cross sections of light nuclear clusters such as deuterons (D), helions $\left({ }^{3} \mathrm{He}\right)$, and tritons $\left({ }^{3} \mathrm{H}\right)[1,2]$. The LHC also brought progress in femtoscopy, the study of the momentum-space correlations of particles emitted in hadronic collisions [5-12]. ${ }^{1}$ These measurements are a source of information on the state produced in heavy-ion collisions [13-21]. A review of future prospects can be found in Ref. [22].

In this paper we consider an interesting feature in the data [23]: the anticorrelation between the source homogeneity volume, probed in femtoscopy, and the coalescence factor of nuclear clusters. This correlation was predicted two decades ago in a seminal work by Scheibl \& Heinz [17]. For a cluster with mass number $A$ and spin $J_{A}$, observed at vanishing transverse momentum $p_{t}=0$ in the collider frame, it is summarized by the relation $[20,23]^{2,3}$

$$
\frac{\mathcal{B}_{A}}{m^{2(A-1)}} \approx \frac{2 J_{A}+1}{2^{A} \sqrt{A}}\left(\frac{m R}{\sqrt{2 \pi}}\right)^{3(1-A)}
$$

Here, the coalescence factor is defined as $\mathcal{B}_{A}=$ $\left(P_{A}^{0} \frac{d N_{A}}{d^{3} P_{A}}\right) /\left(p^{0} \frac{d N}{d^{3} p}\right)^{A}$, where $p^{0} d N / d^{3} p$ is the Lorentz-invariant differential yield for constituent nucleons at $p=P_{A} / A$. The homogeneity volume is parametrized by the HBT radius $R$ $[5-12] .{ }^{4} m \approx 0.94 \mathrm{GeV}$ is the nucleon mass.

\footnotetext{
*kfir.blum@cern.ch
}

Published by the American Physical Society under the terms of the Creative Commons Attribution 4.0 International license. Further distribution of this work must maintain attribution to the author(s) and the published article's title, journal citation, and DOI.
Equation (1) was predicted to apply in the limit that the size parameter $d_{A}$ of the cluster's wave function can be neglected compared with the source homogeneity radius: $d_{A} \ll R$. For small systems with $R \lesssim d_{A}$, Eq. (1) receives a correction via $R^{2} \rightarrow R^{2}+\left(d_{A} / 2\right)^{2}$. At finite $p_{t}$, Ref. [17] suggested that Eq. (1) should be modified by $m \rightarrow m_{t}=\left(m^{2}+p_{t}^{2}\right)^{1 / 2}$.

A comparison of Eq. (1) with LHC data was presented in Ref. [23], which used it to extrapolate measurements in $\mathrm{Pb}-\mathrm{Pb}$ collisions into a prediction of the coalescence factor of $\mathrm{D},{ }^{3} \mathrm{He}$, and ${ }^{3} \mathrm{H}$ in $p-p$ collisions. This extrapolation is nontrivial. The HBT radius characterizing $\mathrm{Pb}-\mathrm{Pb}$ collisions is $R \sim 4 \mathrm{fm}$, compared with $R \sim 1 \mathrm{fm}$ measured in $p$ - $p$ collisions. Thus, Eq. (1) predicts a large increase in $\mathcal{B}_{A}$ going from $\mathrm{Pb}-\mathrm{Pb}$ to $p-p: \mathcal{B}_{3}^{\mathrm{p}-\mathrm{p}} / \mathcal{B}_{3}^{\mathrm{Pb}-\mathrm{Pb}} \sim 4 \times 10^{3}$. Subsequent ALICE measurements [2] in $p-p$ collisions were consistent with this prediction: Eq. (1) appears to work, at least to $O(1)$ accuracy, over orders of magnitude in $\mathcal{B}_{A}$. The question we ask (and answer) in this study is why does it work?

To substantiate this question, note that Ref. [17] derived Eq. (1) by using a number of assumptions and approximations. A simple source model was used to describe the emission of particles produced in hadronic collisions. This model implemented collective flow with a specific velocity profile and a Gaussian density profile, limited to radial symmetry in the transverse direction. By using a saddle-point approximation to evaluate Cooper-Frye integrals [27], Ref. [17] compared their analytic results to a parallel analysis that used the same

\footnotetext{
${ }^{1}$ Also known as Hanbury-Brown-Twiss (HBT) $[3,4]$ analyses.

${ }^{2}$ See also Ref. [16].

${ }^{3}$ See, e.g., Refs. [24-26] for the appearance of a similar formula within a thermodynamic model.

${ }^{4}$ More practical details about the definition of $R$ are given in Sec. IV.
} 
assumptions to calculate HBT parameters [28] and found Eq. (1).

Given this procedure, it is natural to question the theoretical basis for Eq. (1). For example, as noted in Ref. [17], it is unlikely that the source model adopted there can actually describe systems ranging from $\mathrm{Pb}-\mathrm{Pb}$ to $p-p$ in detail. Why then does Eq. (1) work? Can we expect it to remain valid at $p_{t}>0$; at intermediate centrality; and so on?

The outline of our analysis and the main results are as follows: In Sec. II we focus on D formation (Sec. II A) and twoproton correlations (Sec. II B). Using nonrelativistic quantum mechanics (QM) considerations, in idealistic settings ignoring final-state interactions and other real-life complications, we derive a relation between $\mathrm{D}$ formation and two-particle spectra. In Sec. IIC we extend our results to a relativistic formulation. Our main result is Eq. (24), giving $\mathcal{B}_{2}$ as an integral of the two-particle correlation function weighted by the $\mathrm{D}$ probability density. The derivation does not require a detailed model of the particle emission source. In particular, we need not invoke the assumptions and approximations of Refs. [17,28]. Another derivation is shown in Appendix A.

In Sec. III we show that, by adopting the same assumptions, our formalism reproduces Eq. (1) as found in Ref. [17]. ${ }^{5}$ The upshot is that our work makes Eq. (1) a generic prediction. If, above, we argued that the model dependence in Ref. [17] makes it a surprise that Eq. (1) successfully describes systems from $\mathrm{Pb}-\mathrm{Pb}$ to $p$ - $p$, then in light of the discussion in Sec. II it becomes nontrivial to imagine a system for which Eq. (1) would fail. The downside is that Eq. (1) is essentially a kinematical relation and can teach us relatively little about the dynamics of the state produced in heavy-ion collisions. Our analysis bears a connection to (being a less sophisticated version of) Ref. [18], which showed that the number of pion pairs produced in Coulomb bound states is related to the number of free pion pairs at small relative momentum. Our work is also close in spirit to the work by Mrowczynski [14,29-33].

In Sec. IV we consider complications including final-state interactions and source chaoticity (Sec. IV A). We do not address these complications in detail, but show how experimental analyses that take these issues into account can be used to test the coalescence-correlation relation at the cost of some model dependence. In Sec. IV B we generalize our results to $A \geqslant 2$, postponing some details to Appendix B. In Sec. IV C we compare our theoretical results to data. In Sec. IV D we recap the results of Ref. [23], comparing the coalescence-correlation relation with data across systems. While our results are consistent with available measurements, the uncertainties are large. Existing experimental analyses were not geared for a direct comparison of femtoscopy and cluster yields. This lack motivates dedicated experimental work.

We conclude in Sec. V.

\footnotetext{
${ }^{5}$ Apart from the fact that the natural definition we find for $R$ is in the so-called pair rest frame, compared with the longitudinal frame adopted in Ref. [17], and apart from the replacement $m \rightarrow m_{t}$. Please see Sec. III for details.
}

\section{QUANTUM MECHANICS CONSIDERATIONS}

Hadronic collisions produce a high-excitation state (HXS), characterized by a density matrix $\hat{\rho}_{\mathrm{HX}}$. QM allows us to calculate the probability density to find a certain nonrelativistic state in the HXS by projecting that state onto $\hat{\rho}_{\mathrm{HX}}$. In this section we use the QM formalism to derive a relation between $\mathrm{D}$ and two-particle spectra. We then convert to Lorentz-invariant quantities.

We emphasize that the QM formulation we use is far from new. It had been utilized in different guises in many early studies including (as a partial list) Refs. [13,14,16,17,19,28,32-35]. Our discussion in Secs. II A and II B is merely intended to review the derivation of D and particle pair formation, respectively, in the HXS, recalling that the two phenomena stem from building blocks that are closely related on general grounds. Our next step, in Sec. II C, is to explicitly combine the expressions into a direct relation between coalescence and pair spectra, summarized in Eq. (24). This result, as far as we know, is new to the current work.

\section{A. Deuteron formation}

A D at laboratory-frame momentum $P_{d}$ is a two-particle (neutron-proton) bound state $\left|\psi_{P_{d}}\right\rangle$ with wave function

$$
\psi_{P_{d}}\left(x_{1}, x_{2}\right)=e^{i \vec{P}_{d} \vec{X}} \phi_{d}(\vec{r}),
$$

where

$$
\vec{X}=\left(\vec{x}_{1}+\vec{x}_{2}\right) / 2, \quad \vec{r}=\vec{x}_{1}-\vec{x}_{2},
$$

and $\int d^{3} r\left|\phi_{d}(\vec{r})\right|^{2}=1$. The probability density of $\mathrm{D}$ in the $\mathrm{HXS}$ is [13]

$$
\begin{aligned}
\frac{d N_{d}}{d^{3} P_{d}}= & \left\langle\psi_{P_{d}}\left|\hat{\rho}_{\mathrm{HX}}\right| \psi_{P_{d}}\right\rangle \\
= & G_{d} \int d^{3} x_{1} \int d^{3} x_{2} \int d^{3} x_{1}^{\prime} \int d^{3} x_{2}^{\prime} \\
& \times \psi_{P_{d}}^{*}\left(x_{1}^{\prime}, x_{2}^{\prime}\right) \psi_{P_{d}}\left(x_{1}, x_{2}\right) \rho_{2}\left(x_{1}^{\prime}, x_{2}^{\prime} ; x_{1}, x_{2} ; t_{f}\right),
\end{aligned}
$$

where $\rho_{2}\left(x_{1}^{\prime}, x_{2}^{\prime} ; x_{1}, x_{2} ; t_{f}\right)$ is the two-particle reduced HXS density matrix. $G_{d}$ is a dimensionless normalization factor. In this section, for simplicity, we assume the existence of a well-defined freeze-out time $t_{f}$ and consider the HXS density matrix as being specified at the moment $t_{f}$. We emphasize that this simplification is not essential for the derivation, and our main result [Eq. (24) below] holds also if we allow a finite-duration freeze-out window. An alternative derivation that makes this point manifest is given in Appendix A.

It is commonly assumed that the HXS density matrix can be factorized into one-particle density matrices,

$$
\rho_{2}\left(x_{1}^{\prime}, x_{2}^{\prime} ; x_{1}, x_{2} ; t\right)=\rho_{1}\left(x_{1}^{\prime}, x_{1} ; t\right) \rho_{1}\left(x_{2}^{\prime}, x_{2} ; t\right),
$$

that can in turn be described in terms of Wigner densities $f_{1}^{W}$,

$$
\rho_{1}\left(x, x^{\prime} ; t\right)=\int \frac{d^{3} k}{(2 \pi)^{3}} e^{i \vec{k}\left(\vec{x}^{\prime}-\vec{x}\right)} f_{1}^{W}\left(\vec{k}, \frac{\vec{x}+\vec{x}^{\prime}}{2} ; t\right) .
$$


Inserting Eqs. (5) and (6) into Eq. (4), we obtain

$$
\begin{aligned}
\frac{d N_{d}}{d^{3} P_{d}}= & G_{d} \int d^{3} R \int \frac{d^{3} q}{(2 \pi)^{3}} \int d^{3} r \mathcal{D}_{d}(\vec{q}, \vec{r}) \\
& \times f_{1}^{W}\left(\frac{\vec{P}_{d}}{2}+\vec{q}, \vec{R}+\frac{\vec{r}}{2} ; t_{f}\right) f_{1}^{W}\left(\frac{\vec{P}_{d}}{2}-\vec{q}, \vec{R}-\frac{\vec{r}}{2} ; t_{f}\right),
\end{aligned}
$$

where $\mathcal{D}_{d}$ is the Wigner density of the $\mathrm{D}$,

$$
\mathcal{D}_{d}(\vec{q}, \vec{r})=\int d^{3} \zeta e^{-i \vec{q} \vec{\zeta}} \phi_{d}\left(\vec{r}+\frac{\vec{\zeta}}{2}\right) \phi_{d}^{*}\left(\vec{r}-\frac{\vec{\zeta}}{2}\right) .
$$

In terms of the original variables of Eq. (4), $\vec{R}=\left(\vec{x}_{1}+\vec{x}_{1}^{\prime}+\right.$ $\left.\vec{x}_{2}+\vec{x}_{2}^{\prime}\right) / 4$ is the classical center-of-mass coordinate of the two-nucleon system and $\vec{r}=\left(\vec{x}_{1}+\vec{x}_{1}^{\prime}\right) / 2-\left(\vec{x}_{2}+\vec{x}_{2}^{\prime}\right) / 2$ is the classical relative coordinate between the nucleons.

It can be shown that neglecting $\pm \vec{q}$ inside the $f_{1}^{W}$ functions in Eq. (7) is a reasonable approximation, valid to $\sim 10 \%$ accuracy for $\mathrm{Pb}-\mathrm{Pb}$ collisions [17]. With this approximation we can perform the $q$ integration, which gives $\int d^{3} q \mathcal{D}_{d}(\vec{q}, \vec{r})=$ $(2 \pi)^{3}\left|\phi_{d}(\vec{r})\right|^{2}$. Defining

$$
\left|\phi_{d}(\vec{r})\right|^{2}=\int d^{3} k e^{i \vec{k} \vec{r}} \mathcal{D}(\vec{k})
$$

we obtain

$$
\begin{aligned}
\frac{d N_{d}}{d^{3} P_{d}} \approx & G_{d} \int d^{3} q \mathcal{D}(\vec{q}) \int d^{3} R \int d^{3} r e^{i \vec{q} \vec{r}} \\
& \times f_{1}^{W}\left(\frac{\vec{P}_{d}}{2}, \vec{R}+\frac{\vec{r}}{2} ; t_{f}\right) f_{1}^{W}\left(\frac{\vec{P}_{d}}{2}, \vec{R}-\frac{\vec{r}}{2} ; t_{f}\right) .
\end{aligned}
$$

Equation (10) expresses a nonrelativistic QM calculation of the Lorentz noninvariant quantity $d N / d^{3} P_{d}$. In Sec. IIC we return to the problem of connecting this result to the total Lorentz-invariant D yield obtained by integrating over different emission regions in an expanding HXS "fireball."

\section{B. Nucleon pair emission}

Consider a state $\left|\psi_{p_{1}, p_{2}}^{s}\right\rangle$ describing two free propagating protons in a spin-symmetric configuration. Ignoring finalstate interactions (FSIs), the position space representation of $\left|\psi_{p_{1}, p_{2}}^{s}\right\rangle$ is an antisymmetric function of the particle coordinates,

$$
\psi_{p_{1}, p_{2}}^{s}\left(x_{1}, x_{2}\right)=\frac{1}{\sqrt{2}} e^{2 i \vec{P} \vec{X}}\left(e^{i \vec{q} \vec{r} / 2}-e^{-i \vec{q} \vec{r} / 2}\right),
$$

where the average pair momentum and the momentum difference are defined as

$$
\vec{P}=\left(\vec{p}_{1}+\vec{p}_{2}\right) / 2, \quad \vec{q}=\vec{p}_{1}-\vec{p}_{2} .
$$

The probability density associated with $\left|\psi_{p_{1}, p_{2}}^{s}\right\rangle$ can be calculated as $[34,35]$

$$
\begin{aligned}
\frac{d N^{s}}{d^{3} p_{1} d^{3} p_{2}} & =\left\langle\psi_{p_{1}, p_{2}}^{s}\left|\hat{\rho}_{\mathrm{HX}}\right| \psi_{p_{1}, p_{2}}^{s}\right\rangle \\
& =G_{2}^{s} \int d^{3} x_{1} \int d^{3} x_{2} \int d^{3} x_{1}^{\prime} \int d^{3} x_{2}^{\prime} \psi_{p_{1}, p_{2}}^{s *}\left(x_{1}^{\prime}, x_{2}^{\prime}\right) \psi_{p_{1}, p_{2}}^{s}\left(x_{1}, x_{2}\right) \rho_{2}\left(x_{1}^{\prime}, x_{2}^{\prime} ; x_{1}, x_{2} ; t_{f}\right) .
\end{aligned}
$$

Assuming unpolarized isospin-invariant HXS, we use the same $\rho_{2}\left(x_{1}^{\prime}, x_{2}^{\prime} ; x_{1}, x_{2} ; t_{f}\right)$ for the proton-proton and protonneutron reduced density matrix, appearing in Eqs. (13) and (4). $G_{2}^{s}$ is a normalization constant. Inserting Eqs. (5) and (6) into Eq. (13) we obtain

$$
\begin{aligned}
\frac{d N^{s}}{d^{3} p_{1} d^{3} p_{2}}= & G_{2}^{s}\left(\mathcal{A}_{2}\left(p_{1}, p_{2}\right)-\mathcal{F}_{2}(P, q)\right), \\
\mathcal{F}_{2}(P, q)= & \int d^{3} R \int d^{3} r e^{i \vec{q} \vec{r}} f_{1}^{W}\left(\vec{P}, \vec{R}+\frac{\vec{r}}{2} ; t_{f}\right) \\
& \times f_{1}^{W}\left(\vec{P}, \vec{R}-\frac{\vec{r}}{2} ; t_{f}\right), \\
\mathcal{A}_{2}\left(p_{1}, p_{2}\right)= & \int d^{3} x f_{1}^{W}\left(\vec{p}_{1}, \vec{x} ; t_{f}\right) \int d^{3} x f_{1}^{W}\left(\vec{p}_{2}, \vec{x} ; t_{f}\right) .
\end{aligned}
$$

We could express $\mathcal{A}_{2}$ in Eq. (14) in terms of $P$ and $q$, but we keep $p_{1}$, and $p_{2}$ for clarity. The $P, q$ notation is useful for the $\mathcal{F}_{2}$ term, which expresses the QM correlation.

We can repeat the same steps above for the spinantisymmetric state $\left|\psi_{p_{1}, p_{2}}^{a}\right\rangle$, for which the wave function is an symmetric function of the particle coordinates. We find

$$
\frac{d N^{a}}{d^{3} p_{1} d^{3} p_{2}}=G_{2}^{a}\left(\mathcal{A}_{2}\left(p_{1}, p_{2}\right)+\mathcal{F}_{2}(P, q)\right),
$$

with $G_{2}^{a}=G_{2}^{s} / 3$.

\section{Coalescence from two-particle correlations}

Equations (10), (14), and (15) give the number of D and proton pairs, respectively, per differential momentum element when all momenta involved are small. The Lorentz-invariant version of the quantities on the left-hand side (LHS) of these equations are $\gamma_{d} d N_{d} / d^{3} P_{d}$ and $\gamma_{1} \gamma_{2} d N^{s, a} / d^{3} p_{1} d^{3} p_{2}$. Subtleties arise in the computation of the right-hand side (RHS) because, for a relativistically expanding HXS, different parts of the particle emission region are moving relativistically with respect to other parts. This makes the spatial integrations nontrivial [27]. In addition, instead of a homogeneous freezeout time $t_{f}$ we expect a freeze-out surface $t_{f}=t_{f}(\vec{R})$. We now consider these issues. 
Inspecting Eqs. (10) and (14), we can write a differential coalescence-correlation relation

$$
\frac{d}{d^{3} R}\left(\frac{d N_{d}}{d^{3} P_{d}}\right) \approx G_{d} \frac{d}{d^{3} R} \int d^{3} q \mathcal{D}(\vec{q}) \mathcal{F}_{2}\left(\frac{\vec{P}_{d}}{2}, \vec{q}\right) .
$$

The differential presentation reveals model-independence in terms of the details of freeze-out. By either plugging in Eq. (9), or proceeding directly from Eq. (10), we have

$$
\begin{aligned}
\frac{d}{d^{3} R}\left(\frac{d N_{d}}{d^{3} P_{d}}\right)= & G_{d} f_{1}^{W}\left(\frac{\vec{P}_{d}}{2}, \vec{R} ; t_{f}\right) \int d^{3} r\left|\phi_{d}(\vec{r})\right|^{2} f_{1}^{W} \\
& \times\left(\frac{\vec{P}_{d}}{2}, \vec{R}-\vec{r} ; t_{f}\right) .
\end{aligned}
$$

It is natural to regard the RHS of Eq. (17) as a Lorentzinvariant distribution function $f_{d}$. This was done in Ref. [17], which used the Cooper-Frye prescription [27] to make the replacement $\gamma_{d} \int d^{3} R f_{d} \rightarrow(1 / 2 m) \int\left[d^{3} \sigma_{\mu} P_{d}^{\mu}\right] f_{d}$, where $d^{3} \sigma^{\mu}$ is the volume element perpendicular to the HXS relativistic freeze-out surface.

While Ref. [17] (which focused on D formation) arrived at this procedure directly from Eq. (10), the same implementation of freeze-out with respect to the integration over center-of-mass coordinate $\vec{R}$ can be used in integrating the coalescence-correlation relation expressed by Eq. (16). There is no need to specify the details of the freeze-out surface $t_{f}(\vec{R})$ because Eq. (16) relates the pair emissivity and the D emissivity per differential volume element $d^{3} R$ in the HXS. Having noted this point, we can drop the differential $d^{3} R$ in Eq. (16) and consider it as a relation between total D and pair yields.

Let us now make contact with measurements. Experimental collaborations report the (Lorentz-invariant) coalescence factor

$$
\mathcal{B}_{2}(p)=\frac{P_{d}^{0} \frac{d N_{d}}{d^{3} P_{d}}}{\left(p^{0} \frac{d N}{d^{3} p}\right)^{2}},
$$

with $p=P_{d} / 2$ and where $p^{0} \frac{d N}{d^{3} p}$ is the unpolarized proton yield. The two-particle correlation function is constructed as

$$
C_{2}(P, q)=\frac{p_{1}^{0} p_{2}^{0} \frac{d N}{d^{3} p_{1} d^{3} p_{2}}}{\left(p_{1}^{0} \frac{d N}{d^{3} p_{1}}\right)\left(p_{2}^{0} \frac{d N}{d^{3} p_{2}}\right)} .
$$

The numerator on the RHS of Eq. (19) sums together the different spin states of the proton pair. In the denominator, the unpolarized differential yields at $p_{1}$ and $p_{2}$ are obtained by scrambling between proton pairs from different events.

Still provisionally neglecting FSI and other complications (which would be discussed later), Ref. [9] parametrized twoproton correlation measurements in a way that can be put as

$$
C_{2}(P, q)=1-\frac{G_{2}^{s}-G_{2}^{a}}{G_{2}^{s}+G_{2}^{a}} \mathcal{C}_{2}(P, q) .
$$

By examining the $q$ dependence we see that the $\mathcal{C}_{2}$ term in Eq. (20) comes from the $\mathcal{F}_{2}$ term in Eq. (14), while the 1 comes from the $\mathcal{A}_{2}$ term there. More precisely, in the nonrelativistic limit we have

$$
\mathcal{C}_{2}^{\mathrm{PRF}}(|\vec{q}| \ll m)=\frac{\mathcal{F}_{2}}{\mathcal{A}_{2}},
$$

where the superscript PRF instructs us that $q$ in $\mathcal{C}_{2}^{\mathrm{PRF}}$ is defined in the pair center-of-mass frame. In the same limit, Eqs. (16) and (18) show that

$$
\mathcal{B}_{2}(p)=\frac{G_{d}}{G_{2}^{s}+G_{2}^{a}} \frac{2 m}{m^{2} \mathcal{A}_{2}} \int d^{3} q \mathcal{D}(\vec{q}) \mathcal{F}_{2}(\vec{p}, \vec{q}) .
$$

Assuming unpolarized isospin-symmetric HXS [36] we have

$$
\frac{G_{d}}{G_{2}^{s}+G_{2}^{a}}=\frac{3}{3+1} .
$$

Using these conventions and noting that $\gamma_{1} \approx \gamma_{2} \approx \gamma_{d}$ for small $|\vec{q}| \ll m$, we are finally led to the result:

$$
\mathcal{B}_{2}(p) \approx \frac{3}{2 m} \int d^{3} q \mathcal{D}(\vec{q}) \mathcal{C}_{2}^{\mathrm{PRF}}(\vec{p}, \vec{q}) .
$$

Following the discussion around Eq. (16), this result is not limited to nonrelativistic $p$. It is limited to nonrelativistic $|\vec{q}|^{2} \ll m^{2}$, but that is not a real concern because both $\mathcal{C}_{2}$ and $\mathcal{D}$ cutoff at $|\vec{q}| \sim 0.1 \mathrm{~m}$.

We comment that the coalescence factor $\mathcal{B}_{2}(p)$ is defined for on-shell $\mathrm{D}$ with $P_{d}^{2}=4 p^{2} \approx(2 m)^{2}$. Thus, there will actually be no on-shell proton pairs that satisfy $p_{1}^{2}=p_{2}^{2}=m^{2}$ along with $\left(p_{1}+p_{2}\right) / 2=p$ at $q \neq 0$. This problem comes from neglecting corrections of order $\vec{q}^{2} / \mathrm{m}^{2}$ in the derivation of Eq. (24). We can find on-shell proton pairs to construct $\mathcal{C}_{2}^{\mathrm{PRF}}$ by allowing the energy component $P^{0}$ of the $P 4$ vector in Eq. (19) to deviate from $p^{0}$ of Eq. (18), while at the same time enforcing $\vec{P}=\vec{P}_{d} / 2=\vec{p}$. In other words, we let $p$ on the LHS of Eq. (24) denote the four-momentum per nucleon of the on-shell $\mathrm{D}$, and we equate $\vec{p}$ between the LHS and the RHS, but we do not enforce $p^{0}$ on the RHS to match $p^{0}$ on the LHS. Corrections due to this approximation are of order $\vec{q}^{2} / \mathrm{m}^{2}$.

\section{COMPARISON WITH PREVIOUS WORK}

Scheibl \& Heinz [17] used a Gaussian source model (GSM) of the HXS one-particle Wigner densities to calculate coalescence and two-particle correlations (following Ref. [28] on the latter) and expressed the coalescence factor in terms of the HBT radius parameters computed in their model. To obtain analytic expressions, the $\mathrm{D}$ wave function was taken to be Gaussian,

$$
\phi_{d}(\vec{r})=\frac{e^{-\frac{\vec{r}^{2}}{2 d^{2}}}}{\left(\pi d^{2}\right)^{\frac{3}{4}}},
$$

with $d=3.2 \mathrm{fm}$. This leads to

$$
\mathcal{D}(\vec{k})=e^{-\frac{\vec{k}^{2} d^{2}}{4}} .
$$

For the HBT analysis, Ref. [17] used the parameters $R_{\perp}$ and $R_{||}$in terms of which the correlation function in their model is given by

$$
\mathcal{C}_{2}^{\mathrm{PRF}}=e^{-R_{\perp}^{2} \vec{q}_{\perp}^{2}-R_{\|}^{2} \vec{q}_{l}^{2}} \quad(\mathrm{GSM}),
$$

where $\vec{q}_{l}$ is the component of $\vec{q}$ parallel to the beam axis and $\vec{q}_{\perp}$ spans the transverse direction. Plugging these expressions 
for $\mathcal{D}$ and $\mathcal{C}_{2}^{\mathrm{PRF}}$ in Eq. (24) we find ${ }^{6}$

$$
\mathcal{B}_{2}=\frac{3 \pi^{\frac{3}{2}}}{2 m\left(R_{\perp}^{2}+\left(\frac{d}{2}\right)^{2}\right) \sqrt{R_{\|}^{2}+\left(\frac{d}{2}\right)^{2}}} \quad(\mathrm{GSM}) .
$$

This reproduces Eq. (1) and the main result of Ref. [17] [see Eqs. (6.3) and (4.12) there], up to the replacement $m \rightarrow m_{t}=$ $\left(m^{2}+\vec{p}_{t}^{2}\right)^{1 / 2}$. Please note that we have defined $R_{\perp}$ and $R_{\|}$ in the PRF, while Ref. [17] defined these parameters in the YKP frame [28,38-40] which is offset by a transverse boost compared with the PRF.

Mrowczynski discussed the connection between coalescence and two-particle correlations in a series of papers [14,29-33]. This program resulted in a QM sum rule of the neutron-proton correlation function, which was proposed to give the $\mathrm{D}$ coalescence factor as a $q$ integral on the correlation function [33]. The power of this idea was in that there was no need to correct the measured correlation function for longor short-range final-state interactions: the sum rule should apply directly to the observable correlation. In practice, this suggestion fails, apparently because the $q$ integral proposed in Ref. [33] receives contributions from large- $q$ regions in the integration.

In comparison to the sum rule of Refs. [32,33], Eq. (24) is less ambitious. The correlation function entering Eq. (24) does need to be corrected for final-state interactions, because it assumes a kinetic picture where an HXS density matrix can be defined and projected into propagating particles. Equation (24) also invokes assumptions such as isospin symmetry and smoothness for the HXS freeze-out surface. In return, however, the RHS of Eq. (24) receives no contributions from large- $q$ modes because $\mathcal{D}(\vec{q})$ in the integrand constrains the support to the small- $q$ region, $|\vec{q}| \lesssim 0.1 \mathrm{~m}$.

A QM derivation of the coalescence factor using a specific one-dimensional Gaussian source model was given in Ref. [19]. This derivation agrees with Eq. (28) up to the replacement $m \rightarrow p^{0}=m \gamma_{d}$.

\section{REAL-LIFE COMPLICATIONS, $A>2$ CLUSTERS, AND COMPARING WITH DATA}

Equation (24) is idealistic. In practice we cannot pull out a directly measured correlation function $\mathcal{C}_{2}$, plug into Eq. (24), and calculate $\mathcal{B}_{2}$. Two main complications preventing direct implementation of Eq. (24) are that (i) long-lived resonances, decaying outside of the freeze-out surface of the HXS, distort the correlations, and (ii) long-range Coulomb and short-range strong nuclear FSI cause the two-particle wave function to differ from the plane-wave form. For proton pairs, FSI actually dominate the correlation function, meaning that the QM statistics contribution must be extracted indirectly as a subleading contribution to the actual observable $\mathcal{C}_{2}$. To make things more difficult, different spin states exhibit different short-range FSI.

We will not address the complications above in detail in this paper, deferring such refinements to future work. Instead,

\footnotetext{
${ }^{6}$ See also Refs. [16,37].
}

we build on femtoscopy data analyses that explicitly treat items (i) and (ii). The price we pay is to introduce model dependence, which enters via an assumed simple analytic form for the correlation function. Our procedure and results are explained in the next sections.

\section{A. The chaoticity parameter $\lambda$}

The GSM assumed in Refs. [17,19,28] predicts not only the shape, but also the normalization of $\mathcal{C}_{2}$ : it predicts $\mathcal{C}_{2}^{\mathrm{PRF}}(\vec{q} \rightarrow$ $0)=1$. In reality, measurements show $\mathcal{C}_{2}^{\mathrm{PRF}}(\vec{q} \rightarrow 0) \rightarrow \lambda<$ 1 , where $\lambda$ is known as the chaoticity (or intercept) parameter [41,42]. In HBT analyses of pions, $\lambda<1$ follows from the fact that a sizable fraction of the pions come from the decay of long-lived resonances, leading to a non-Gaussian contribution to $\mathcal{C}_{2}$ that is concentrated at very small $|\vec{q}|$ and cannot be resolved experimentally [41]. In HBT analyses of proton pairs, hyperons are the resonant contamination $[11,43,44]$. Since strong FSI between $p \Lambda$ and $p p$ are crucial in shaping the $p \Lambda$ and $p p$ correlation functions, studies $[9,11,43,44]$ separate the $p \Lambda \rightarrow p p$ and genuine $p p$ contributions entering the observed $p p$ correlation into different terms, which are fit in a combined analysis. In Refs. $[9,11]$, separate chaoticity parameters $\lambda_{p p}, \lambda_{p \Lambda}$ were assigned to the genuine $p p$ pairs and the pairs coming from $p \Lambda \rightarrow p p$. The value of $\lambda$ defined in this way could reflect intrinsic departures of the source functions from Gaussianity.

In Ref. [28] (and many other analyses in the literature), $\lambda$ was introduced as a free parameter. Thus, it did not enter into the coalescence-HBT correspondence of Ref. [17]. However, Eq. (24) shows that $\mathcal{B}_{2}$ is directly proportional to a $q$ moment of $\mathcal{C}_{2}^{\mathrm{PRF}}$. If we adopt the Gaussian form together with the $\lambda$ modification as an empirical description of $\mathcal{C}_{2}$,

$$
\mathcal{C}_{2}^{\mathrm{PRF}}=\lambda e^{-R_{\perp}^{2} \vec{q}_{\perp}^{2}-R_{\|} \vec{q}_{l}^{2}} \quad(\mathrm{GSM}, \text { chaoticity } \lambda),
$$

then $\mathcal{B}_{2}$ should match Eq. (28) simply multiplied by the experimentally deduced value of $\lambda$ :

$$
\mathcal{B}_{2}=\frac{3 \pi^{\frac{3}{2}} \lambda}{2 m\left(R_{\perp}^{2}+\left(\frac{d}{2}\right)^{2}\right) \sqrt{R_{\|}^{2}+\left(\frac{d}{2}\right)^{2}}} \quad(\text { GSM, chaoticity } \lambda) .
$$

$$
\text { B. } A \geqslant 2
$$

Equation (24) can be generalized to clusters with $A \geqslant 2$. Assuming an $(A-1)$-dimensional symmetric Gaussian form for the cluster's relative coordinate wave function, and assuming that the $A$-particle correlation function can be decomposed as a product of two-particle Gaussian correlators described by the same HBT radii $R_{\perp}$ and $R_{\|}$and chaoticity $\lambda$, then the analog of Eq. (30) is

$$
\frac{\mathcal{B}_{A}}{m^{2(A-1)}}=\lambda^{\frac{A}{2}} \frac{2 J_{A}+1}{2^{A} \sqrt{A}}\left[\frac{(2 \pi)^{\frac{3}{2}}}{m^{3}\left(R_{\perp}^{2}+\left(\frac{d_{A}}{2}\right)^{2}\right) \sqrt{R_{\|}^{2}+\left(\frac{d_{A}}{2}\right)^{2}}}\right]^{A-1} .
$$

The definitions of the cluster wave function and its size parameter $d_{A}$, used in Eq. (31), are given in Appendix B. 


\section{Comparing with data}

Experimental collaborations often report the results of HBT analyses in terms of empirical fit parameters $R$ and $\lambda$ [5-12], assuming Eq. (29) and accounting explicitly for the spin symmetry of the pair wave function and for the distortion due to FSI $[18,34,45]$. To compare our theoretical results with data, we will therefore use Eqs. (30) and (31). We further take the extra simplification of a one-dimensional HBT parametrization with $R_{\perp}=R_{\|}=R$.

Pion, kaon, and proton femtoscopy results in $\mathrm{Pb}-\mathrm{Pb}$ collisions were reported in Refs. [9,11]. Results for proton and kaon femtoscopy in $p$ - $p$ collisions were given in Refs. [12] and [6], respectively. The kaon results are of potential use because Ref. [9] showed compatible results for the parameters $R$ and $\lambda$ obtained in proton and kaon correlations at the same $m_{t}$. Using these HBT analyses we can calculate the RHS of Eqs. (30) and (31), and compare with experimental data on the production of light nuclei [1,2].

\section{Pb-Pb collisions}

The top two panels in Fig. 1 summarize experimental results for $R$ and $\lambda$ in central $(0 \%-10 \%) \mathrm{Pb}-\mathrm{Pb}$ collisions at $\sqrt{s}=2.76 \mathrm{TeV}$ [9]. ${ }^{7}$ The bottom two panels show $R$ and $\lambda$ obtained in intermediate centrality $(30 \%-50 \%)$ data. For $R$, we show the average values found for $p p$ and $\bar{p} \bar{p}$ pairs. The uncertainties are mostly systematic, and the width of the band neglects the statistical uncertainty. For $\lambda$, we show the sum $\lambda_{p p}+\lambda_{p \Lambda}$, take the average of the systematic uncertainty, and average the result between particles and antiparticles. ${ }^{8}$

Plugging these values of $R, \lambda$ into the RHS of Eq. (30), we obtain a prediction for $\mathcal{B}_{2}$. The result for $(0 \%-10 \%)$ centrality events is shown by the blue shaded band in the topmost panel of Fig. 2. The uncertainty of the theory prediction was obtained by using the lower value for $\lambda$ and the upper value for $R$ to calculate the lower value of the predicted $\mathcal{B}_{2}$, and vice versa. An experimental measurement of $\mathcal{B}_{2}$ [1] is shown in the same plot as a gray band. We can also compare the data with the theoretical prediction of Ref. [17]; this is done in the second-from-top panel of Fig. 2. In the bottom two panels of Fig. 2 we repeat the analysis by using the intermediate centrality $(30 \%-50 \%) \mathrm{HBT}$ parameters, compared with $\mathcal{B}_{2}$ data corresponding to events at $(20 \%-40 \%)$ and $(40 \%-60 \%)$ centrality events. ${ }^{9}$

\footnotetext{
${ }^{7}$ Useful details can be found in Tables 7.4-7.9 in Ref. [11].

${ }^{8}$ The reason to use the sum of $\lambda_{p p}+\lambda_{p \Lambda}$, and not just $\lambda_{p p}$, is that we are interested in using Eq. (30), which assumes the same single-particle spectrum normalization in the definition of $\mathcal{C}_{2}$ and $\mathcal{B}_{2}$. However, the single-particle spectrum entering the denominator of $\mathcal{B}_{2}$ in the experimental analysis includes only the prompt contribution, while the denominator in the $\mathcal{C}_{2}$ experimental analysis with $p p$ and $p \Lambda$ terms explicitly separated includes both prompt and secondary protons.

${ }^{9}$ Note that the analysis of Ref. [17] was restricted to radially symmetric HXS in the plane transverse to the beam axis. It should not, in principle, be valid for intermediate centrality.
}
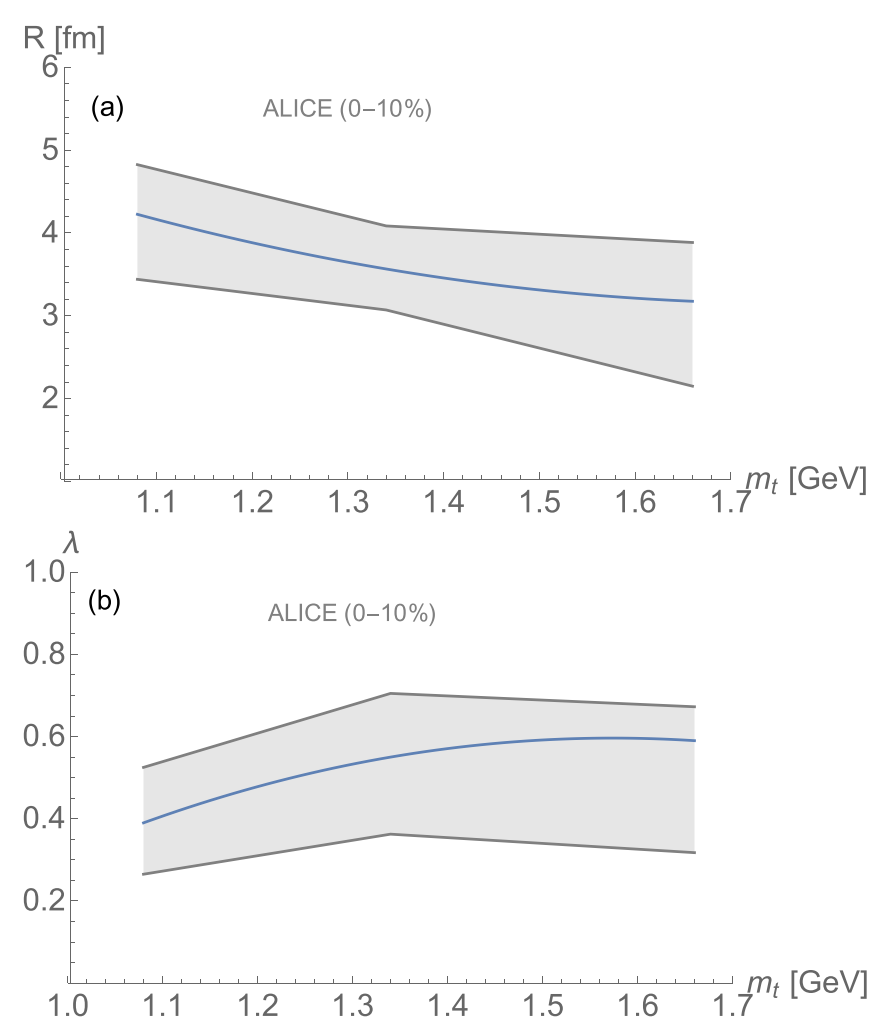

$\mathrm{R}[\mathrm{fm}]$

(c)
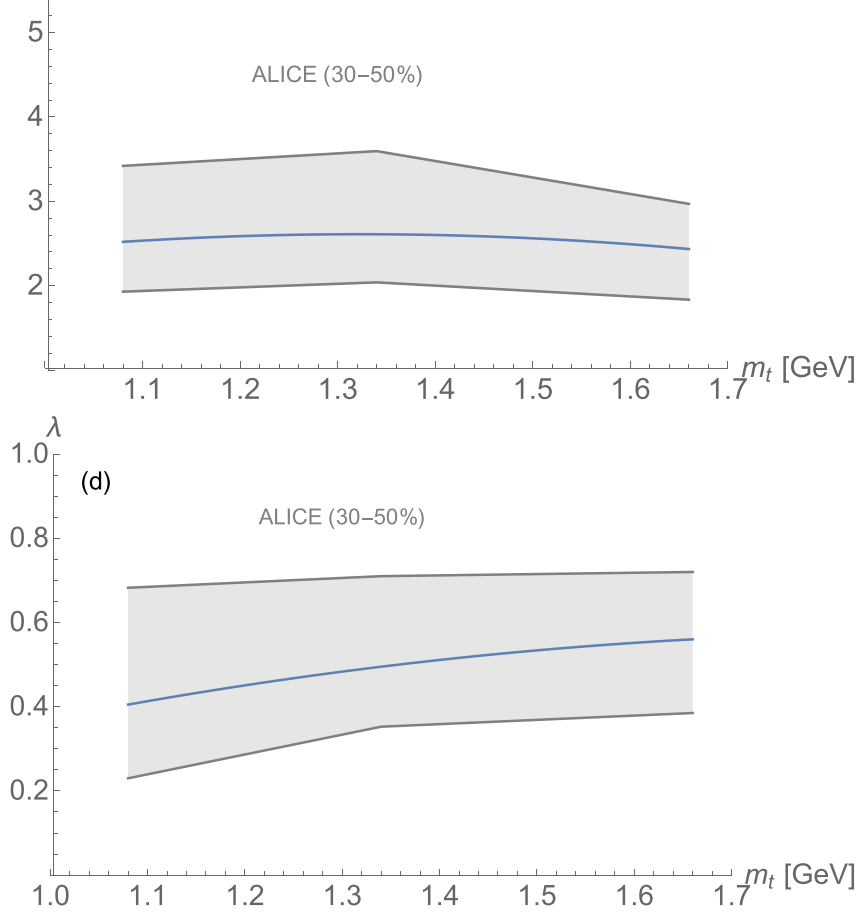

FIG. 1. Experimental fit results for the one-dimensional HBT radius $R$ and $\lambda$ parameters, extracted from correlations of $p p, p \Lambda$, and their antiparticles in central $[0 \%-10 \%$; panels (a) and (b)] and intermediate centrality (30\%-50\%; [panels (c) and (d)] $\mathrm{Pb}-\mathrm{Pb}$ collisions at $\sqrt{s}=2.76 \mathrm{TeV}[9,11]$. 

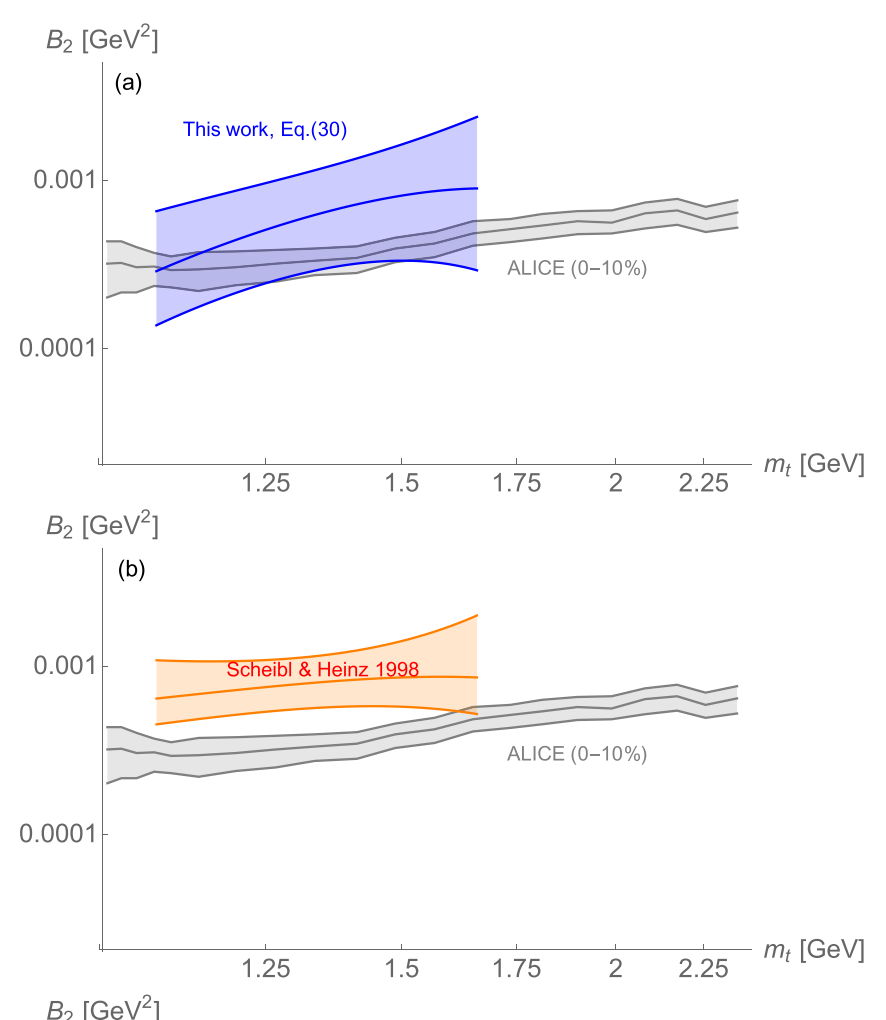

(c)

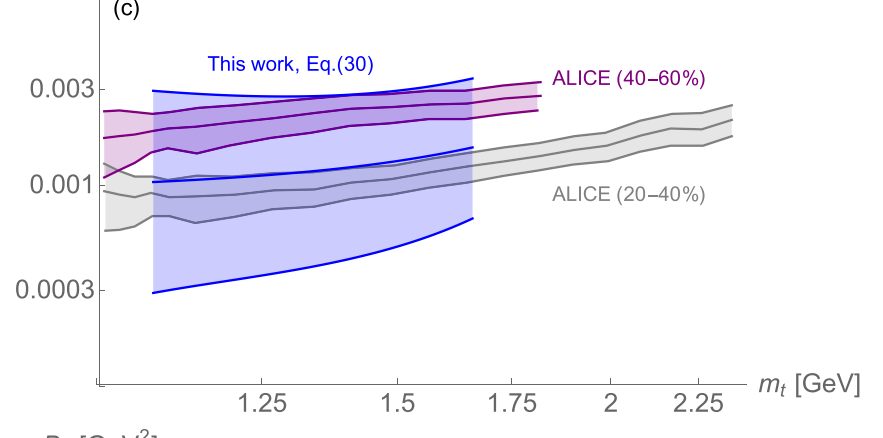

$B_{2}\left[\mathrm{GeV}^{2}\right]$

(d)

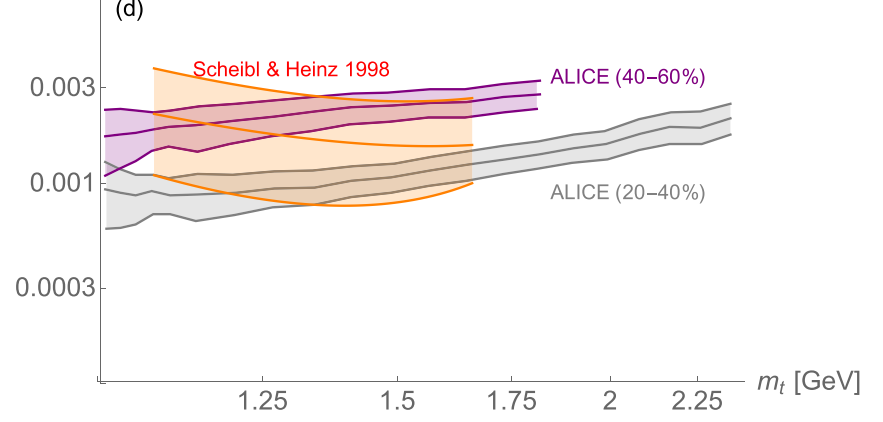

FIG. 2. (a), (b) Experimental results for $\mathcal{B}_{2}$ from central $(0 \%-10 \%) \mathrm{PbPb}$ collisions at $\sqrt{s}=2.76 \mathrm{TeV}$ [1], shown by gray band, compared with Eq. (30) derived here (blue band) and to the prediction of Ref. [17] (orange band). The coalescence calculation uses the experimentally extracted HBT $R$ and $\lambda$ parameters shown in Fig. 1. (c), (d) Experimental values of $\mathcal{B}_{2}$ from two intermediate centrality classes, $(20 \%-40 \%)$ and $(40 \%-60 \%)$, and the theoretical prediction calculated using HBT data from events at (30\%-50\%).
In Fig. 3 we consider experimental results for $\mathcal{B}_{3}[1]$ from centrality classes $(0 \%-20 \%)$ and $(20 \%-80 \%)$, shown in the top two and bottom two panels, respectively.

\section{2. $p$-p collisions}

Reference [12] reported $R \approx 1.14_{-0.02}^{+0.07}$ fm (comparable statistic and systematic uncertainties were added in quadrature) in a combined analysis of $p p, p \Lambda$, and other hyperon correlation data from $\sqrt{s}=7 \mathrm{TeV} p-p$ collisions at pair average momentum corresponding to $m_{t}=(1.2-1.6) \mathrm{GeV}$. The analysis in this work effectively assumed $\lambda=1$. However, in an analysis that allowed $\lambda$ to vary as a free parameter, kaon correlations were found to give $\lambda \sim 0.5$ at $m_{t}=1.4 \mathrm{GeV}$, along with $R \approx 0.8 \pm 0.3 \mathrm{fm}$ [6]. This is of potential interest because Ref. [9] demonstrated HBT parameters that were the same, within measurement uncertainties, for kaon and proton final states at the same $m_{t}$.

Using $R \approx 1.14_{-0.02}^{+0.07} \mathrm{fm}$ as found in the $p p$ analysis [12], Eqs. (30) predicts $\mathcal{B}_{2}=10^{-2} \times(0.8-0.9) \lambda \mathrm{GeV}^{2}$. Using, instead, $R \approx 0.8 \pm 0.3 \mathrm{fm}$ as found from kaon correlations [6], Eq. (30) predicts $\mathcal{B}_{2}=10^{-2} \times(0.9-1.4) \lambda \mathrm{GeV}^{2}$. These predictions can be compared with light cluster data from Ref. [2], which found the experimental result $\mathcal{B}_{2}^{\exp } \approx 10^{-2} \times$ (1.6-2.2) $\mathrm{GeV}^{2}$ at $m_{t} \approx 1.4 \mathrm{GeV}$.

Using $R \approx 1.14_{-0.02}^{+0.07}$ fm [12], Eq. (31) predicts $\mathcal{B}_{3}=$ $10^{-4} \times(2.1-2.8) \lambda^{\frac{3}{2}} \mathrm{GeV}^{4}$. For $R \approx 0.8 \pm 0.3$ fm [6], Eq. (31) predicts $\mathcal{B}_{3}=10^{-4} \times(3.1-23) \lambda^{\frac{3}{2}} \mathrm{GeV}^{4}$. The experimental result [2] is $\mathcal{B}_{3}^{\exp } \approx 10^{-4} \times(1-3) \mathrm{GeV}^{4}$ at $m_{t} \approx$ (1.1-1.4) GeV.

\section{Discussion: $\mathcal{B}_{A}$ vs $R$, coalescence across systems}

Measurement uncertainties on the HBT $R$ and $\lambda$ parameters lead to large uncertainties on our theoretical prediction of $\mathcal{B}_{2}$ and $\mathcal{B}_{3}$, derived from Eqs. (30) and (31). Part of this uncertainty is due to our crude treatment of the data. For example, our uncertainty estimate on $\mathcal{B}_{2}$ and $\mathcal{B}_{3}$ in the left panels of Figs. 2 and 3 added together the effects of the systematic measurement uncertainties on $R$ and $\lambda$. As a result, while Eqs. (30) and (31) are consistent with the data, there is much room to improve the analysis. The coalescence-correlation correspondence motivates an experimental reassessment of the data presented in Refs. $[6,9,12]$ and $[1,2]$, aiming at a joint analysis of HBT and cluster yields in events sharing the same $p_{t}$ and centrality classes.

Before we conclude, in Fig. 4 we take a broader look at the data-theory comparison by considering the $\mathcal{B}_{A}-R$ (anti)correlation across different systems [23]. In Fig. 4, the gray shaded band shows the theoretical prediction for $\mathcal{B}_{2}$ (top) and $\mathcal{B}_{3}$ (bottom), calculated as function of $R$ using Eqs. (30) and (31). The calculation uses an estimate of the experimentally measured value of $\lambda$. To define the upper edge of the bands, we interpolate between $\lambda=\{1,0.7,0.7\}$ defined at $R=\{0.85,2.5,5\}$. To define the lower edge we interpolate between $\lambda=\{0.5,0.3,0.3\}$ defined at $R=\{0.85,2.5,5\}$. This range of $\lambda$ is roughly consistent with the experimental results found in Refs. $[6,9,12]$. The red horizontal bands in Fig. 4 show the $(0 \%-10 \%)$ (for $\left.\mathcal{B}_{2}\right)$ and $(0 \%-20 \%)$ (for $\mathcal{B}_{3}$ ) 

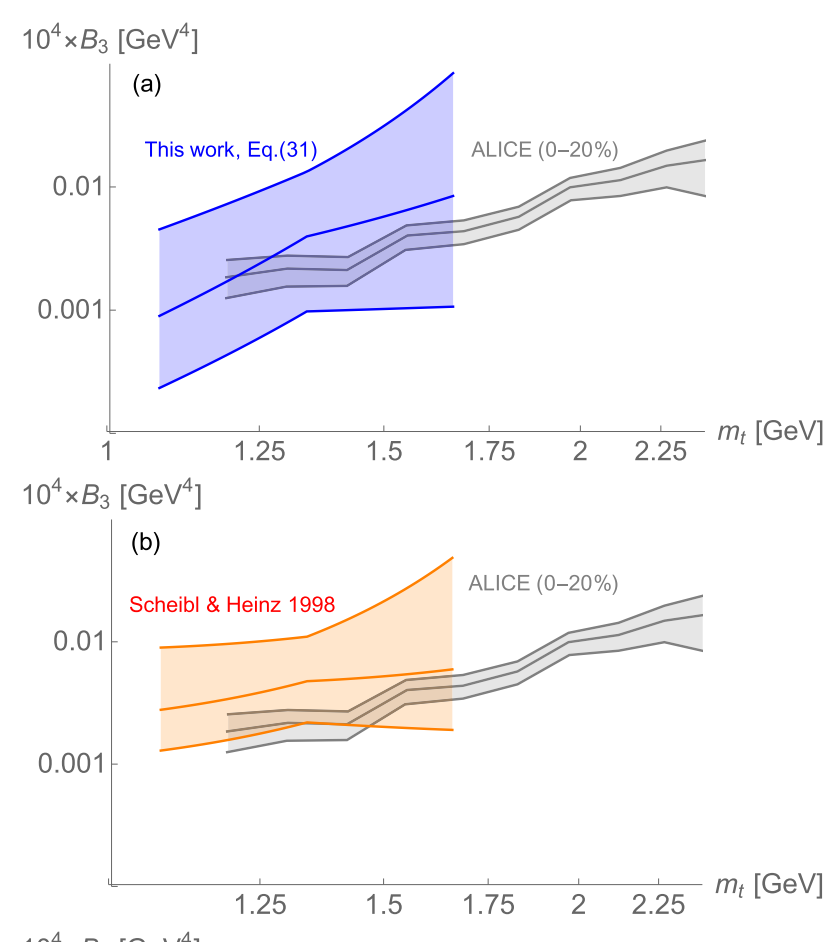

$10^{4} \times B_{3}\left[\mathrm{GeV}^{4}\right]$
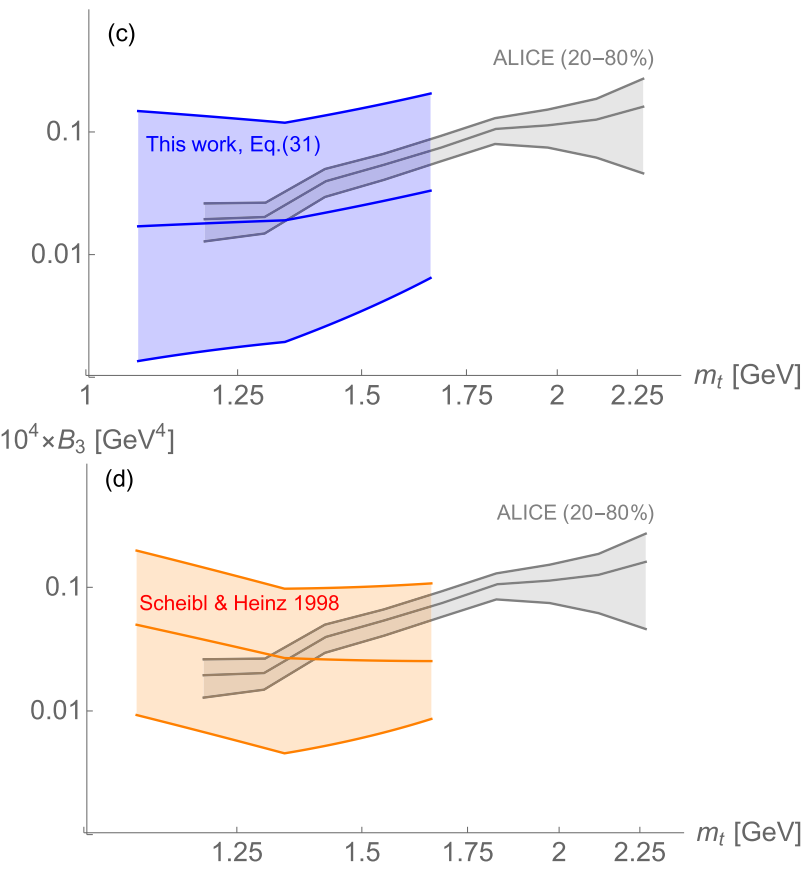

FIG. 3. (a), (b) Experimental results for $\mathcal{B}_{3}$ from central $(0 \%-$ $20 \%) \mathrm{PbPb}$ collisions at $\sqrt{s}=2.76 \mathrm{TeV}$ [1], shown by gray band, compared with Eq. (31) derived here (blue band) and to the prediction of Ref. [17] (orange band). (c), (d) Experimental values of $\mathcal{B}_{3}$ from the centrality class $(20 \%-80 \%)$, and the theoretical prediction calculated using HBT data from events at (30\%-50\%).

coalescence factor measurements for $\mathrm{Pb}-\mathrm{Pb}$. Each of the three red bands corresponds to a different bin in $m_{t}$, among the three bins shown in Ref. [9]. The blue horizontal bands show the result for the $(20 \%-40 \%)$ (for $\left.\mathcal{B}_{2}\right)$ and $\left(20 \%-80 \%\right.$ ) (for $\mathcal{B}_{3}$ )
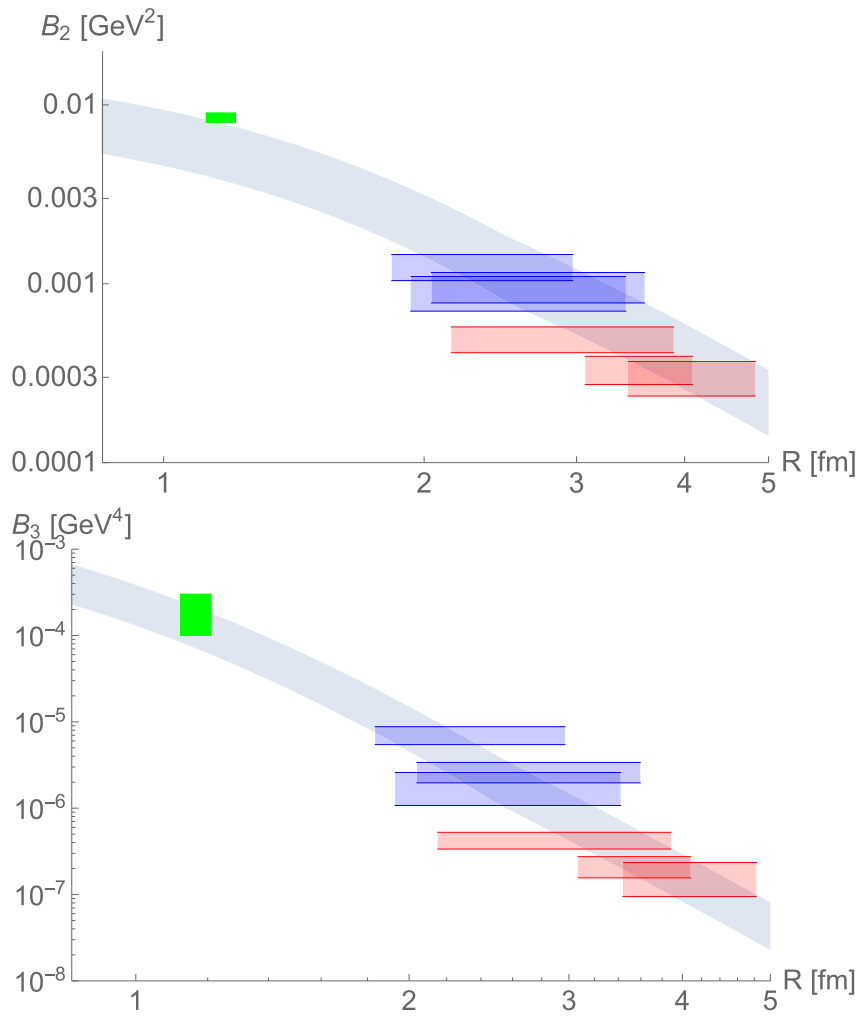

FIG. 4. Summary of data. (top) $\mathcal{B}_{2}$ vs $R$. (bottom) $\mathcal{B}_{3}$ vs $R$.

events, respectively. The green band shows the result for $p-p$ collisions [2].

\section{CONCLUSIONS}

We considered the relation between nuclear cluster formation (defined via a coalescence factor $\mathcal{B}_{A}$ ) and two-particle correlation measurements [known as femtoscopy or HanburyBrown-Twiss (HBT) analyses, with two-particle correlation function $\mathcal{C}_{2}$ ] in hadronic collisions. Scheibl \& Heinz [17] derived a theoretical result, Eq. (1), equating $\mathcal{B}_{A}$ to inverse powers of the source homogeneity radius $R$ measured in HBT analyses. Equation (1) is consistent with LHC data over several orders of magnitude in $\mathcal{B}_{A}$, albeit with large uncertainties [23]. Reference [17] based their derivation of Eq. (1) on a specific, simplified model of collective flow. This model is unlikely to actually represent in detail the dynamics in different systems ranging from $\mathrm{Pb}-\mathrm{Pb}$ to $p-p$. The question we addressed to ourselves was, therefore, why does Eq. (1) work?

Using an idealized quantum-mechanical (QM) framework, we derived a direct integral relation between the coalescence factor and the two-particle correlation function. Our main result is Eq. (24), which gives $\mathcal{B}_{2}$ as an integral of $\mathcal{C}_{2}$ weighted by the $\mathrm{D}$ probability density. The derivation does not require a detailed model of the particle emission source. In particular, we need not invoke the assumptions and approximations of Ref. [17]. If we specialize to the assumptions in Ref. [17], our formula essentially reproduces Eq. (1). Importantly, Eq. (1) also obtains under more general circumstances if the two- 
particle correlation function can be approximately described empirically by a Gaussian form, as commonly used in experimental HBT studies.

While our theoretical results are consistent with currently available measurements, the uncertainties are large. Existing experimental analyses were not geared for a direct comparison of femtoscopy and cluster yields. No HBT analysis precisely overlaps, in terms of, e.g., $p_{t}$ and centrality binning, with cluster yield measurements. The recent study in Ref. [20] (see also Ref. [46]) proposed to bypass this gap by replacing the HBT part in the coalescence-correlation comparison with multiplicity measurements that correlate with the HBT scales. We suggest, instead, that the coalescence-correlation relation offers a fundamental probe of the (generally defined) coalescence model, justifying dedicated experimental work aiming to test the relation directly.

\section{ACKNOWLEDGMENTS}

We thank Francesca Bellini, Alexander Kalweit, and Urs Wiedemann for discussions and JinJin Pan and Kenny $\mathrm{Ng}$ for ongoing collaboration on data analysis related to this work. We are grateful to Ulrich Heinz for discussions and especially for helping us find our way in the literature on coalescence and HBT in heavy-ion collisions. K.B. is incumbent of the Dewey David Stone and Harry Levine career development chair. The work of K.B. and M.T. was supported by Grant 1937/12 from the I-CORE program of the Planning and Budgeting Committee and the Israel Science Foundation and by Grant 1507/16 from the Israel Science Foundation.

\section{APPENDIX A: COALESCENCE FROM CORRELATION FUNCTIONS: KINETIC THEORY}

Here we give another derivation of Eq. (24). The starting point of our analysis is equivalent to Eq. (3.12) of Ref. [17], derived in Ref. [15].

We assume that the two-particle source can be factorized as a product of one-particle source terms. The production rate of deuterons (D) at momentum $P_{d}$, per four-dimensional volume in the source region parametrized by $\mathrm{D}$ formation coordinates $R$, is given by

$$
\begin{aligned}
\frac{d}{d^{4} R} \frac{d N_{d}}{d^{3} P_{d}}= & \frac{3 \times 2}{(2 \pi)^{3}} \int \frac{d^{3} r d^{3} Q}{(2 \pi)^{3}} \mathcal{D}_{d}(\vec{Q}, \vec{r}) \\
& \times f\left(R_{+} ; Q_{+}\right) \Gamma_{\text {free }}\left(R_{-} ; Q_{-}^{*}\right),
\end{aligned}
$$

where the factor 3 is due to the deuteron spin and the factor 2 is due to exchange of proton and neutron. $\Gamma_{\text {free }}$ indicates the production rate of free nucleons. We have $Q_{+}+Q_{-}^{*}=$ $P_{d}$, and we take $Q_{-}^{*}$ slightly off-shell to ensure momentum conservation. For small $\vec{Q}$, we can approximate

$$
\begin{aligned}
\frac{d}{d^{4} R} \frac{d N_{d}}{d^{3} P_{d}} \approx & \frac{3 \times 2}{(2 \pi)^{3}} \int d^{3} r\left|\phi_{d}(\vec{r})\right|^{2} \\
& \times f\left(R_{+} ; P_{d} / 2\right) \Gamma_{\text {free }}\left(R_{-} ; P_{d} / 2\right) .
\end{aligned}
$$

It is convenient to consider the coalescence problem in the D rest frame (DRF). In the DRF, we define the source function $S$ as

$$
S(x)=\frac{m \Gamma_{\text {free }}(x)}{(2 \pi)^{3}},
$$

such that the free nucleon distribution function is given by

$$
f(y)=\frac{(2 \pi)^{3}}{m} \int_{-\infty}^{y_{0}} d t S(t, \vec{y}) .
$$

For small $|\vec{Q}|^{2} \ll m^{2}$, the constituent nuclei energies are $\approx m$ in the DRF, so the Lorentz invariant $\mathrm{D}$ yield is

$$
\begin{aligned}
\left(E_{d} \frac{d N_{d}}{d^{3} P_{d}}\right)^{\mathrm{DRF}} \approx & \frac{2 m}{m^{2}} 3 \times 2(2 \pi)^{3} \int d^{4} R \frac{1}{2} \int d^{4} r\left|\phi_{d}(\vec{r})\right|^{2} \\
& \times S\left(R^{0}-t, \vec{R}-\frac{\vec{r}}{2} ; m\right) S\left(R^{0}, \vec{R}+\frac{\vec{r}}{2} ; m\right) .
\end{aligned}
$$

Now, consider the two-point correlation function $\mathcal{C}_{2}(P, q)$. $\mathcal{C}_{2}(q, P)$ depends on the frame and we take the pair centerof-mass frame (PRF). For clarity, we use the symbol $\mathcal{C}_{2}^{\mathrm{PRF}}$ to define the two-point function in this frame. Under the same source factorization assumption we considered for the coalescence problem, we have [28]

$$
\mathcal{C}_{2}^{\mathrm{PRF}}(P, q)=\frac{4 \int d^{4} R \int d^{4} r S\left(R+\frac{r}{2} ; P\right) S\left(R-\frac{r}{2} ; P\right) e^{i q \cdot r}}{\left(E \frac{d N}{d^{3} P}\right)^{2}},
$$

where the factor 4 comes from the spin combinations.

Comparing Eqs. (A5) and (A6), and using Eq. (9), we reproduce Eq. (24).

\section{APPENDIX B: CLUSTER WAVE FUNCTION}

We consider the cluster internal wave function to be a symmetric Gaussian function of the normalized Jacobi coordinates $\vec{\xi}_{n}, n=1, \ldots, A-1$,

$$
\phi_{A}\left(\vec{\xi}_{1}, \ldots, \vec{\xi}_{A-1}\right)=\frac{\exp \left(-\frac{\sum_{i=1}^{A-1} \vec{\xi}_{i}^{2}}{2 d_{A}^{2}}\right)}{A^{\frac{3}{4}}\left(\pi d_{A}^{2}\right)^{\frac{3(A-1)}{4}}}
$$

where [47]

$$
\vec{\xi}_{n}=\frac{n}{\sqrt{n^{2}+n}}\left(\vec{r}_{n+1}-\frac{1}{n} \sum_{m=1}^{n} \vec{r}_{m}\right)
$$

and where $\vec{r}_{m}, m=1, \ldots, A$ are the Cartesian constituent nucleon coordinates. The size parameter $d_{A}$ is related to the cluster rms charge radius via [17,20,36]

$$
r_{\mathrm{rms}}^{2}=\frac{3(A-1)}{2 A} d_{A}^{2}
$$


[1] J. Adam et al. (ALICE Collaboration), Phys. Rev. C 93, 024917 (2016).

[2] S. Acharya et al. (ALICE Collaboration), Phys. Rev. C 97, 024615 (2018).

[3] R. H. Brown and R. Q. Twiss, Nature (London) 178, 1046 (1956).

[4] R. H. Brown and R. Q. Twiss, Nature (London) 177, 27 (1956).

[5] K. Aamodt et al. (ALICE Collaboration), Phys. Rev. D 82, 052001 (2010).

[6] B. Abelev et al. (ALICE Collaboration), Phys. Rev. D 87, 052016 (2013).

[7] B. B. Abelev et al. (ALICE Collaboration), Phys. Rev. C 89, 024911 (2014).

[8] A. Kisiel, M. Gałażyn, and P. Bożek, Phys. Rev. C 90, 064914 (2014).

[9] J. Adam et al. (ALICE Collaboration), Phys. Rev. C 92, 054908 (2015).

[10] J. Adam et al. (ALICE Collaboration), Phys. Rev. C 91, 034906 (2015).

[11] M. P. Szymanski, Ph.D. thesis, CICQM, 2016 (unpublished).

[12] S. Acharya et al. (ALICE Collaboration), Phys. Rev. C 99, 024001 (2019).

[13] H. Sato and K. Yazaki, Phys. Lett. B 98, 153 (1981).

[14] S. Mrowczynski, J. Phys. G: Nucl. Phys. 13, 1089 (1987).

[15] P. Danielewicz and P. Schuck, Phys. Lett. B 274, 268 (1992).

[16] W. J. Llope et al., Phys. Rev. C 52, 2004 (1995).

[17] R. Scheibl and U. W. Heinz, Phys. Rev. C 59, 1585 (1999).

[18] R. Lednicky, Phys. Part. Nucl. 40, 307 (2009).

[19] S. Mrowczynski, Acta Phys. Pol., B 48, 707 (2017).

[20] F. Bellini and A. P. Kalweit, arXiv:1807.05894.

[21] P. Braun-Munzinger and B. Dönigus arXiv:1809.04681.

[22] Z. Citron et al., arXiv:1812.06772.

[23] K. Blum, K. C. Y. Ng, R. Sato, and M. Takimoto, Phys. Rev. D 96, 103021 (2017).
[24] A. Mekjian, Phys. Rev. Lett. 38, 640 (1977).

[25] A. Z. Mekjian, Nucl. Phys. A 312, 491 (1978).

[26] S. Das Gupta and A. Z. Mekjian, Phys. Rep. 72, 131 (1981).

[27] F. Cooper and G. Frye, Phys. Rev. D 10, 186 (1974).

[28] S. Chapman, J. R. Nix, and U. W. Heinz, Phys. Rev. C 52, 2694 (1995).

[29] S. Mrowczynski, Phys. Lett. B 248, 459 (1990).

[30] S. Mrowczynski, Phys. Lett. B 277, 43 (1992).

[31] S. Mrowczynski, Phys. Lett. B 308, 216 (1993).

[32] S. Mrowczynski, Phys. Lett. B 345, 393 (1995).

[33] R. Maj and S. Mrowczynski, Phys. Rev. C 71, 044905 (2005).

[34] S. E. Koonin, Phys. Lett. B 70, 43 (1977).

[35] S. Pratt, T. Csörgõ, and J. Zimanyi, Phys. Rev. C 42, 2646 (1990).

[36] R. Mattiello, H. Sorge, H. Stöcker, and W. Greiner, Phys. Rev. C 55, 1443 (1997).

[37] M. J. Murray and B. Holzer, Phys. Rev. C 63, 054901 (2001).

[38] F. B. Yano and S. E. Koonin, Phys. Lett. B 78, 556 (1978).

[39] M. I. Podgoretsky, Sov. J. Nucl. Phys. 37, 272 (1983) [Yad. Fiz. 37, 455 (1983)].

[40] Y. F. Wu, U. W. Heinz, B. Tomasik, and U. A. Wiedemann, Eur. Phys. J. C 1, 599 (1998).

[41] U. A. Wiedemann and U. W. Heinz, Phys. Rev. C 56, 3265 (1997).

[42] S. V. Akkelin, R. Lednicky, and Yu. M. Sinyukov, Phys. Rev. C 65, 064904 (2002).

[43] F.-Q. Wang, Phys. Rev. C 60, 067901 (1999).

[44] F.-q. Wang and S. Pratt, Phys. Rev. Lett. 83, 3138 (1999).

[45] R. Lednicky and V. L. Lyuboshits, Sov. J. Nucl. Phys. 35, 770 (1982) [Yad. Fiz. 35, 1316 (1981)].

[46] K.-J. Sun, C. M. Ko, and B. Dönigus, Phys. Lett. B 792, 132 (2019).

[47] A. Shebeko, P. Papakonstantinou, and E. Mavrommatis, Eur. Phys. J. A 27, 143 (2006). 